\title{
Spatially Adaptive Algorithm for Impulse Noise Removal from Color Images
}

\author{
Vitaly Kober ${ }^{1}$, Mikhail Mozerov², and Josué Álvarez-Borrego ${ }^{3}$ \\ ${ }^{1}$ Department of Computer Sciences, Division of Applied Physics \\ CICESE, Ensenada, B.C. 22860, Mexico, \\ vkober@cicese.mx \\ ${ }^{2}$ Laboratory of Digital Optics, Institute for Information Transmission Problems \\ Bolshoi Karetnii 19, 101447 Moscow, Russia \\ mozer@itp.ru \\ ${ }^{3}$ Optics Department, Division of Applied Physics \\ CICESE, Ensenada, B.C. 22860, Mexico \\ josue@cicese.mx
}

\begin{abstract}
An effective method for removal impulse noise in corrupted color images is proposed. The method consists of two steps. Outliers are first detected using spatial relationships between the color image components. Then the detected noise pixels are replaced with the output of the vector median filter over a local spatially connected area excluding the outliers, while noise-free pixels are left unaltered. Simulation results in a test color image show a superior performance of the proposed filtering algorithm comparing with the conventional vector median filter. The comparisons are made using a mean square error, a mean absolute error, and a subjective human visual error criteria.
\end{abstract}

\section{Introduction}

Color images are often corrupted by impulse noise due to a noise sensor or channel transmission errors. The major objective of impulse noise removal is to suppress the noise while preserving the image details. Color images can be considered as twodimensional three channel signals. So, monochrome image processing techniques such as median and, in general, order statistics filters [1-4] demonstrating good ability in the removal of impulse noise can be applied to each color component plane. However, such component-wise noise removal does not give desirable results because the output values may be with possible chromaticity shifts. Therefore, it is desirable to employ the dependence between the color components. Recently an effective nonlinear vector filter called as vector median filter (VMF) [5] was proposed. The VMF and its variants [6] represent ones of the most popular approaches for noise removal in color images. However, because these approaches are typically implemented uniformly across a color image, they also tend to modify pixels that are undisturbed by noise. Moreover, they are prone to edge jitter when the percentage of 
impulse noise is large. Consequently, the effective removal of impulses is often at the expense of blurred and distorted features. Recently nonlinear filters for monochrome images with a signal-dependent shape of the moving window have been proposed [7]. In this paper, we utilize the approach for suppressing the impulse noise in highly corrupted color images. First outliers are detected using spatial relationships between the color components. Then the detected noise pixels are replaced with the output of the VMF computed over a local spatially connected area excluding the outliers from the area. In the case of independent channel impulse noise, the proposed detector greatly reduces the miss probability of impulse noise. The performance of the proposed filter is compared with that of the conventional VMF algorithm.

The presentation is organized as follows. In Section 2, we present a novel efficient algorithm for detection of noise impulses. A modified vector median filter using the proposed detector is also described. In Section 3, with the help of computer simulation we test the performance of the conventional and proposed filter. Section 4 summarizes our conclusions.

\section{Spatially Adaptive Algorithm for Detection and Removal Impulse Noise}

In impulse noise models, corrupted pixels are often replaced with values near to the maximum and minimum of the dynamic range of a signal. In our experiments, we consider a similar model in which a noisy pixel can take a random value either from sub-ranges of the maximum or the minimum values with a given probability. The distribution of impulse noise in the sub-ranges can be arbitrary. To detect impulse noise in a color image, we use the concept of a spatially connected neighborhood. An underlying assumption is as follows: image pixels geometrically close to each other belong to the same structure or detail. The spatially connected neighborhood is defined as a subset of pixels $\left\{v_{n, m}\right\}$ of a moving window, which are spatially connected with the central pixel of the window, and whose values deviate from the value of the central pixel $v_{k, l}$ at most predetermined quantities $-\varepsilon_{v}$ and $+\varepsilon_{v}$ [7]:

$$
\operatorname{CEV}\left(v_{k, l}\right)=\operatorname{CON}\left(\left\{v_{n, m}: v_{k, l}-\varepsilon_{v} \leq v_{n, m} \leq v_{k, l}+\varepsilon_{v}\right\}\right) .
$$

The size and shape of a spatially connected neighborhood are dependent on characteristics of image data and on parameters, which define measures of homogeneity of pixel sets. So the spatially connected neighborhood is a spatially connected region constructed for each pixel, and it consists of all the spatially connected pixels, which satisfy a property of similarity with the central pixel.

The vector median filter replaces the color vector of each pixel by the vector median value. However, the VMF is often implemented uniformly across a color image. This leads to undesired smoothing of image details, which are uncorrupted by impulse noise. Therefore, the quality of the filtering depends on an impulse noise detector. The detector must decrease the probabilities of impulse noise miss and false detection. 
In other words, it should detect as much as possible noisy pixels, while the false detection should be as less as possible to preserve image details. We propose to detect outliers with the help of spatial relations between the color components. We assume that a spatially connected region corrupted with impulse noise is relatively small comparing to details of the image. Therefore, the impulsive noise can be detected by checking the size of its region. If the size is less than a given threshold value, say M, impulse noise is detected. Obviously, such a detector omits impulses with the size greater than $M$. The probability of occurrence of a four-connected noise cluster of the size $M$ in a moving window can be computed using the addition formula of probabilities. The noise cluster occurs simultaneously with one of the mutually exclusive events $H_{l}, \ldots, H_{N}$. Here $H_{k}$ is the event denoting that there is a noise cluster of the size exactly $\mathrm{M}$ noise impulses surrounded by uncorrupted image pixels. The probability of occurrence of a noise cluster of the size $M$ at a given image pixel is given as

$$
\operatorname{Pr}(M)=\sum_{k=1}^{N} \operatorname{Pr}\left(H_{k}\right)
$$

where the probability of the event $H_{k}$ is $\operatorname{Pr}\left(H_{k}\right)=P^{M}(1-P)^{E_{k}(M)}, E_{k}(M)$ is the number of surrounded uncorrupted image pixels. Taking into account that some of the probabilities $\operatorname{Pr}\left(H_{k}\right)$ are equal, the Eq.(2) is computationally simplified to

$$
\operatorname{Pr}(M)=P^{M} \sum_{k=1}^{K(M)} C_{k}(M)(1-P)^{E_{k}(M)},
$$

where $K(M), C_{k}(M), E_{k}(M)$ are coefficients determined from the geometry (binary region of support) of the cluster of noise.

Table 1. Coefficients for calculating the probability of impulsive clusters

\begin{tabular}{|c|c|c|c|c|}
\hline Size of cluster M & $K(M)$ & $k$ & $C_{k}(M)$ & $E_{k}(M)$ \\
\hline 1 & 1 & 1 & 1 & 4 \\
\hline 2 & 1 & 1 & 4 & 6 \\
\hline 3 & 2 & 1 & 12 & 7 \\
& & 2 & 6 & 8 \\
\hline 4 & 3 & 1 & 36 & 8 \\
& & 2 & 32 & 9 \\
& & 3 & 8 & 10 \\
\hline 5 & 5 & 1 & 5 & 8 \\
& & 2 & 100 & 9 \\
& & 3 & 140 & 10 \\
& & 4 & 60 & 11 \\
& & 5 & 10 & 12 \\
\hline
\end{tabular}

For a given image pixel, $K(M)$ is the number of groups, each of them contains $C_{k}(M)$ events $H_{k}$ with the equal probabilities $\operatorname{Pr}\left(H_{k}\right), \mathrm{k}=1, \ldots K(M)$. For example, the number of groups with $M=2$ is $K(2)=1$, and the number of surrounding four-connected 
uncorrupted pixels is $E_{l}(M)=6$. The number of the events is $C_{l}(M)=4$ (four possible variants of the noise cluster on the grid including the given pixel). With the help of Table 1 and Eq. (3), the probability of occurrence of a four-connected impulse noise cluster of the size $M$ can be easily calculated. Table 2 presents the probability of occurrence of impulse cluster of size $M$ versus the probability of impulse noise on a rectangular grid. We see that when the probability of impulse noise is high, the occurrence of impulse cluster is very likely.

Table 2. The probability of occurrence of impulse clusters of the size M versus the probability $\mathrm{P}$ of impulse noise.

\begin{tabular}{|c|c|c|c|}
\hline \multirow{2}{*}{$\mathrm{M}$} & \multicolumn{3}{|c|}{ Probability of impulse noise } \\
\cline { 2 - 4 } & $\mathrm{P}=0.01$ & $\mathrm{P}=0.1$ & $\mathrm{P}=0.2$ \\
\hline 0 & 0.99 & 0.9 & 0.8 \\
\hline 1 & $5.6 \times 10^{-3}$ & $6.5 \times 10^{-2}$ & $8.2 \times 10^{-2}$ \\
\hline 2 & $3.7 \times 10^{-4}$ & $2.1 \times 10^{-2}$ & $4.2 \times 10^{-2}$ \\
\hline 3 & $1.7 \times 10^{-5}$ & $8.3 \times 10^{-3}$ & $2.8 \times 10^{-2}$ \\
\hline 4 & $7 \times 10^{-7}$ & $3 \times 10^{-3}$ & $1.8 \times 10^{-2}$ \\
\hline 5 & $2.8 \times 10^{-8}$ & $1.1 \times 10^{-3}$ & $1.1 \times 10^{-2}$ \\
\hline
\end{tabular}

Here we provided the coefficients for $M \leq 5$. In a similar manner, the coefficients for greater sizes of noise clusters can be calculated.

Suppose that impulsive noise is independent in $L$ signal channels. The probability of occurrence of a noise cluster of the size $M$ at a given image pixel can be written as

$$
\operatorname{Pr}(M)=\left(P^{L}\right)^{M} \sum_{k=1}^{K(M)} C_{k}(M)\left(1-P^{L}\right)^{E_{k}(M)},
$$

For a color image $(L=3)$, the probability of impulse noise with $M=1$ and $P=0.1$ becomes 0.000996 (compare to 0.065 for $\mathrm{L}=1$ ). We see that the probability of multichannel impulse noise greatly decreases when the number of channels increases. The algorithm of impulse noise detection in a color image is given as follows. First, we construct spatially connected neighborhoods in the RGB channels, independently. The parameters spatially connected neighborhoods in the channels are chosen on the base either a priori or measured information about the spread of the signal to be preserved.

Let ICON and UCON be two sets obtained as intersection and union of the regions of supports of the spatially connected channel neighborhoods $\mathrm{CON}_{\mathrm{R}}, \mathrm{CON}_{\mathrm{G}}, \mathrm{CON}_{\mathrm{B}}$, respectively. If the number of elements in ICON is small, then at least in one channel there exists impulse noise. If the size of UCON is large, then a detected impulse is probably in one channel. If the both sets are small, impulse noise is in three channels. However, the probability of this event is very small. Finally, for the moving window of $3 \times 3$ pixels we use the following threshold values for the sets: if the size of ICON $\leq$ 1 and $\mathrm{UCON} \leq 1$, the central pixel is corrupted in three channels; if the size of ICON $\leq 2$ and the difference between two sizes $\geq 3$, then the central pixel is corrupted in one 
or two channels; if the size of ICON $\geq 2$ and the difference $\leq 2$, the central pixel is not corrupted and there is a high local signal variation in the channels.

Finally, the detected impulse noise is replaced with the output of the VMF computed over a local spatially connected area excluding the outliers. The conventional VMF is defined as follows. For a set of $N$ vectors in the RGB color space $S \in\left(\mathbf{x}_{1}, \mathbf{x}_{2}, \ldots \mathbf{x}_{N}\right), \mathbf{x}_{n}=\left(R_{n}, G_{n}, B_{n}\right)$ with a vector norm $\|\mathbf{X}\|_{L}$, the vector median filter is given by

$$
\mathbf{x}_{V M}=\left(R_{V M}, G_{V M}, B_{V M}\right), \mathbf{x}_{V M} \in S
$$

with

$$
\sum_{\mathrm{n}=1}^{\mathrm{N}}\left\|\mathbf{x}_{V M}-\mathbf{x}_{n}\right\|_{\mathrm{L}} \leq \sum_{\mathrm{n}=1}^{\mathrm{N}}\left\|\mathbf{x}_{a}-\mathbf{x}_{n}\right\|_{L}, \quad \forall \mathbf{x}_{a} \in S .
$$

This operation selects such a vector in the moving window, which minimizes the sum of the distances to the other $\mathrm{N}-1$ vectors regarding the L-norm. We suggest to find the median value among the vectors belonging only to the set of spatially connected neighborhoods with the region of support UCON excluding corrupted pixels. However, if the size of UCON is small, for noise filtering a small region surrounding UCON is used. The proposed algorithm is extension of the algorithm [7] to color images, and it can be written as

$$
\hat{v}_{n, m}=\left\{\begin{array}{lr}
v_{n, m}, & \text { if } \operatorname{SIZE}(I C O N) \geq T h_{-} I C O N \\
\operatorname{VMF}\left(v_{n, m}\{U C O N\}-v_{n, m}\{\operatorname{ICON}\}\right), & \text { if } \operatorname{SIZE}(U C O N) \geq T h_{-} U C O N, \\
\operatorname{VMF}\left(v_{n, m}\{U C O N\}-v_{n, m}\{U C O N\}\right), & \text { otherwise }
\end{array}\right.
$$

where Th_ICON and Th_UCON are threshold values of outlier detection for the sets ICON and UCON, respectively; "-" denotes the set difference operationvvvv; $v_{n, m}\{\mathrm{~S}\}$ is the subset of pixels of the moving window with the region of support $\mathrm{S} ; \overline{U C O N}$ is a small region surrounding UCON. The algorithm starts from the first line of Eq. (6).

\section{Computer Experiments}

Signal processing of a color image degraded due to impulse noise is of interest in a variety of tasks. Computer experiments are carried out to illustrate and compare the performance of conventional and proposed algorithms. We are interested in answering how well, relative to the other filters, does each perform in terms of noise removal and preservation of fine structures. However, it is difficult to define an error criterion to accurately quantify image distortion. In this paper, we will base our comparisons on the mean square error (MSE), the mean absolute error (MAE), and a subjective visual criterion. The empirical normalized mean square error is given by 


$$
M S E=\frac{\sum_{n=1}^{N} \sum_{m=1}^{M} \sum_{k=1}^{3}\left|v_{n, m, k}-\hat{v}_{n, m, k}\right|^{2}}{\sum_{n=1}^{N} \sum_{m=1}^{M} \sum_{k=1}^{3} v_{n, m, k}^{2}},
$$

where $\left\{v_{n, m, k}\right\}$ and $\left\{\hat{v}_{n, m, k}\right\}$ are the original image and its estimate (filtered image), respectively. In our simulations, $\mathrm{N}=320, \mathrm{M}=200$ (320x200 image resolution), and each pixel has 256 levels of quantization. The empirical normalized mean absolute error is defined as:

$$
M A E=\frac{\sum_{n=1}^{N} \sum_{m=1}^{M} \sum_{k=1}^{3}\left|v_{n, m, k}-\hat{v}_{n, m, k}\right|}{\sum_{n=1}^{N} \sum_{m=1}^{M} \sum_{k=1}^{3}\left|v_{n, m, k}\right|} .
$$

The use of these error measures allows us to compare the performance of each filter. Fig. 1(a) shows a test color image degraded due to impulse noise. The probability of independent noise impulse occurrence is 0.07 in each color channel. This means that the total noise probability is $P_{R G B}=1-(1-P)^{3}=1-0.93^{3} \approx 0.2$. In computer simulation, the values of impulses were set to $0-15$ or $240-255$ with equal probability. Table 3 shows the errors under the MSE and MAE criteria for the VMF and the proposed filter. The size of the moving window is $3 \times 3$. The value $\varepsilon_{v}$ to construct spatially connected channel neighborhoods is equal to 10 . The threshold values are taken as $T h \_I C O N=1$ and Th_UCON=4. The first two rows in the table show the errors after filtering of the original image without noise. We see that in this case the conventional VMF has a worse performance comparing with the proposed algorithm. Fig 1 (b, c) show the filtered images obtained from the noise image in Fig. 1 (a) with the conventional VMF and the proposed filter, respectively. The proposed filter using the spatial pixel connectivity has a strong ability in impulse noise suppression and a very good preservation of fine structures and details. The visual comparison of the filtered images in Fig. 1 (b) and 1 (c) shows that the filtered image with the VMF is much smoother than the output image after filtering with proposed method.

Table 3. Impulse noise suppression with different filters

\begin{tabular}{|c|c|c|}
\hline \multirow{2}{*}{ Type of Filters } & \multicolumn{2}{|c|}{ Measured Errors } \\
\cline { 2 - 3 } & MSE & MAE \\
\hline VMF 3x3 (WN) & 0.0173 & 0.0802 \\
\hline $\begin{array}{c}\text { Proposed algorithm } \\
\text { (WN) }\end{array}$ & 0.0014 & 0.0056 \\
\hline Noisy image & 0.0897 & 0.1338 \\
\hline VMF 3x3 & 0.0197 & 0.0887 \\
\hline Proposed algorithm & 0.0087 & 0.0319 \\
\hline
\end{tabular}




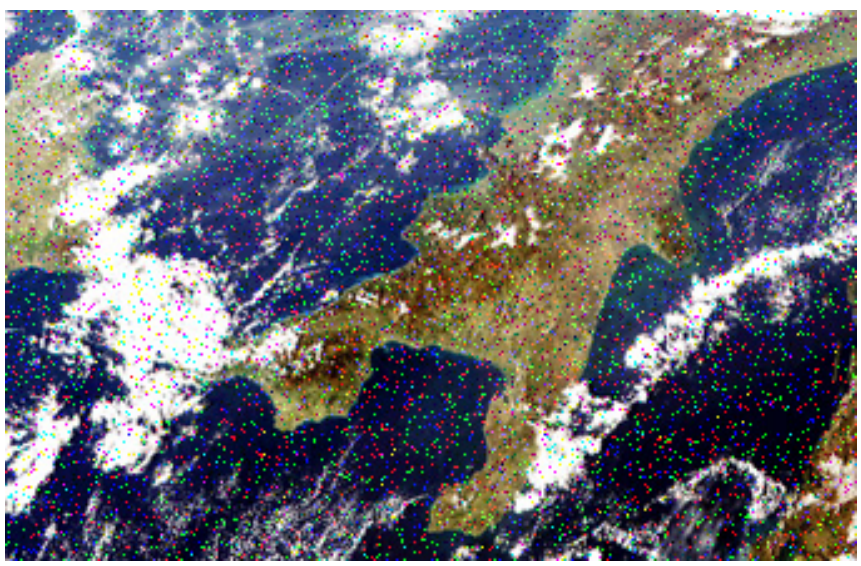

Fig. 1. (a) Noisy color image

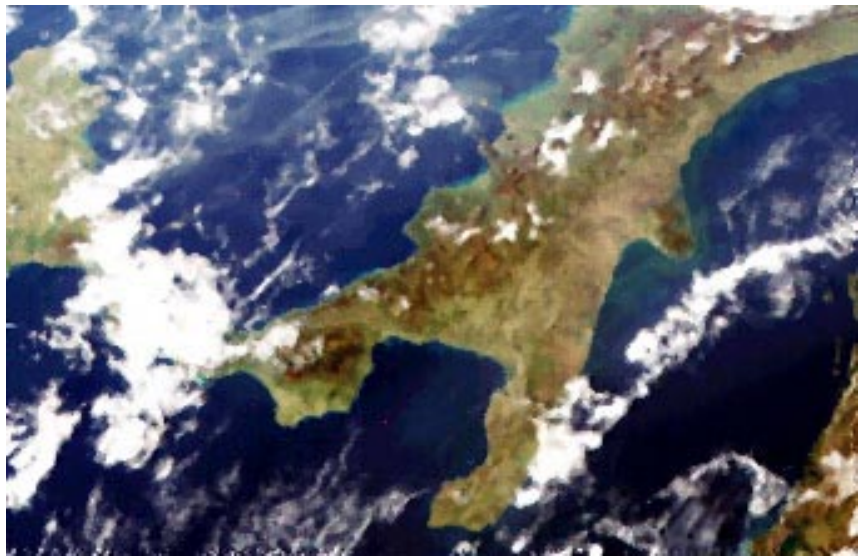

Fig. 1. (b) Filtered image by VMF

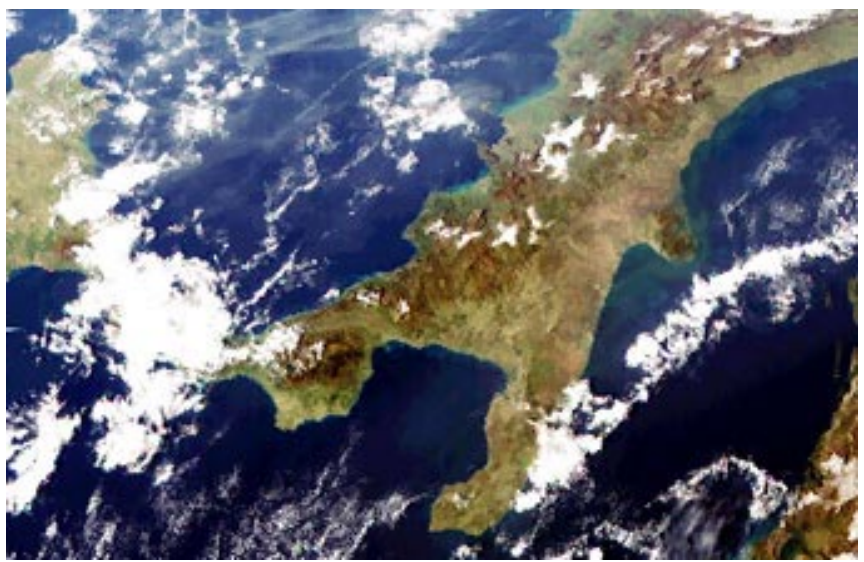

Fig. 1. (c) Filtered image by the proposed algorithm 


\section{Conclusion}

In this paper, we have presented a new algorithm for detection and suppression of impulse noise in color images. The filter utilizes an explicit use of spatial relations between color image elements. When the input color image is degraded due impulse noise, extensive testing has shown that the proposed spatially adaptive vector median filter outperforms the conventional vector median filter in terms of the mean square error, the mean absolute error, and a subjective visual criterion.

Acknowledgment. This work was supported by the grant 36077-A from CONACYT.

\section{References}

1. Arce G.R., Gallagher N.C., and Nodes T., Median filters: Theory and applications, in Advances in Computer Vision and Image Processing, Huang, T., Ed. Greenwich, CT: JAI (1986).

2. Pitas I. and Venetsanopoulos A.N., Nonlinear digital filters. Principles and applications, Kluwer Academic Publishers, Boston (1990).

3. Tsekeridou S., Kotropoulos C., Pitas I., Adaptive order statistic filters for the removal of noise from corrupted images, Optical Engineering, Vol. 37, (1998), 2798-2815.

4. Abreu E., Linghtstone M., Mitra S.K., and Arakawa K., A new efficient approach for the removal of impulse noise from highly corrupted images, IEEE Trans. on Image Processing, Vol. 2, No. 6, (1993), 1012-1025.

5. Astola j., Haavisto P., and Neuvo Y., Vector median filter," Processing of the IEEE, Vol. 78, (1990), 678-689.

6. Vardavoulia M.I., Andreadis I., Tsalides Ph., A new vector median filter for colour image processing, Pattern Recognition Letters, Vol. 22, (2001), 675-689.

7. Kober V., Mozerov M., Alvarez-Borrego J., Nonlinear filters with spatially connected neighborhoods, Optical Engineering, Vol. 40, No. 6, (2001), 971-983. 